\title{
Is Sitting Time a Strong Predictor of Weight Gain?
}

\author{
Elin Kolle • Ulf Ekelund
}

Published online: 9 December 2012

(C) Springer Science+Business Media New York 2012

\begin{abstract}
The current review aimed to examine the evidence on prospective associations between sedentary behavior (i.e., sitting time) and weight gain. Prospective studies published between January 2010 and August 2012 were identified from searches in the Medline databases. In total 13 studies (seven in adolescents and six in adults) examining the prospective association between sedentary behavior and any measure of weight gain met inclusion criteria. In adolescents, mixed evidence was observed for a positive association between sedentary behavior at baseline and weight gain at follow-up. In adults, there was insufficient evidence that sedentary time at baseline was a strong predictor of weight gain at follow-up. The majority of the included studies used self- or parent-reported sedentary time which can be affected by social desirability and recall bias. Marked heterogeneity in study populations, exposure and outcome measures precluded a quantitative meta-analysis. Failure to appropriately adjust the results for baseline measures of the outcome particularly in the studies in children and adolescents may partly explain the observations. Therefore, firm conclusions whether prolonged sitting time predict weight gain in young people and adults is currently not possible. High-quality studies using repeated objective measures of sedentary behavior is warranted to establish a causal association.
\end{abstract}

Keywords Sedentary behavior · Sitting $\cdot$ Longitudinal studies · Weight gain · Adult · Youth · Obesity · Overweight

\author{
E. Kolle $(\bowtie) \cdot$ U. Ekelund \\ Department of Sports Medicine, Norwegian School of Sport \\ Sciences, P.O. Box 4014, Ullevål Stadion, \\ 0806 Oslo, Norway \\ e-mail: elin.kolle@nih.no \\ U. Ekelund \\ Medical Research Council Epidemiology Unit, \\ Institute of Metabolic Science, Cambridge, UK
}

\section{Introduction}

The increase in the prevalence of overweight and obesity observed globally during the last 20 to 30 years may be associated with reduced energy-expenditure due to physical activity in everyday life. In today's society people spend the majority of the day being inactive or in sedentary pursuits, including transport, occupation and during leisure time. Recent estimates using objective measures of physical activity and sedentary time in population representative samples suggest that no more than 3-4 percent of the awake time is spent in physical activity of moderate to vigorous intensity, whereas 60 percent of the waking hours are spent in sedentary pursuits $[1,2]$. Sedentary behavior has been defined as activities characterized by sitting or reclining position and requiring an energy expenditure $<1.5$ METs (multiples of the basal metabolic rate) [3]. Light intensity activities are those done while standing, but that requires less than 3.0 METs [4]. It includes activities such as slow walking, cooking food and washing the dishes.

Accumulating evidence suggests that sedentary behavior, usually defined as sitting time or time watching TV is associated with increased cardiovascular risk [5], all-cause mortality [6,7], and a variety of physiological and psychological problems independent of confounding factors in adults. A recent prospective study reported that independent of age, sex, body mass index (BMI), self-rated health, level of physical activity, baseline cardiovascular disease and diabetes, and other confounders, longer hours of sitting were dose-dependently associated with greater risk of all-cause mortality across an average of 2.8 years of follow-up [8]. It has also been estimated that for every hour of TV time after age 25 years, Australian adults can expect a 22 minute reduction in their life expectancy [9]. A meta-analysis of prospective cohort studies suggested that that TV viewing was consistently associated with higher risk of type 2 diabetes, cardiovascular disease, and all-cause mortality [10]. 
Despite the accumulating observational evidence suggesting that sitting time and TV viewing are long term predictors of morbidity and mortality these results should be interpreted cautiously. First, sitting time and TV viewing time are difficult to assess by self-report, and they are prone to reporting bias [11]. Second, sitting and TV viewing time may be a proxy for a generally unhealthy lifestyle including a higher consumption of high-energy dense foods and drinks. Although some of the cited studies have tried to control for diet intake as a confounding variable, this habit is notoriously difficult to accurately assess in any observational study. Therefore, we cannot rule out the possibility that the observed results are explained by residual confounding due to poorly measured or unmeasured confounders [11]. Third, both waist circumference and BMI have shown to partly mediate or attenuate the association between TV viewing and CVD mortality [5] and CVD risk factors [12]. Adiposity should therefore be considered as a confounder or mediator when examining the association between sedentary time and health outcomes [11]. Forth, it appears obvious that total time spent sedentary is the reciprocal of overall physical activity energy expenditure. Studies that have adjusted their analyses for physical activity have only controlled for a subcomponent, e.g., time spent in moderate and vigorous intensity activity or leisure time activity, of overall activity. It could therefore be hypothesized that the observed associations between sedentary time and health outcomes would be similar but in opposite direction (i.e., inverse) when employing a measure of overall physical activity. Indeed, objectively measured physical activity energy expenditure and total body movement are associated with increased risk of death [13, 14].

Several studies have also examined the relationship between sedentary time (e.g., total sitting time, time spent watching TV and other screen based behaviors) and obesity and gain in body weight in both the young population and adults. To date, the majority of evidence on the associations between sedentary time and obesity is based on self-report and only a few studies have relied on objective assessment of sedentary time. Nevertheless, most studies have reported cross-sectional associations between time spent watching TV and overweight or obesity $[15,16]$. A recent study in 8233 Australian women reported an association between sitting time and weight in overweight and obese women [17]. In the young population, a review of cross-sectional studies found sedentary behaviors to be positively associated with weight status [18]. The majority of studies examining the association between sitting time and obesity have been cross-sectional, limiting the ability to make inferences about causality and temporal sequence. It is still unclear whether increased sitting time predisposes individuals to gain weight, or whether weight gain predisposes individuals to sit more. Chinapaw et al. [19] published a systematic review summarizing the relationship between young peoples' sedentary behavior and BMI or other indicators of fat mass from longitudinal studies up to 2010. Thorp et al. (2011) conducted a systematic review of prospective observational studies investigating associations between sedentary behavior and subsequent health outcome in adults, including sedentary behaviors effect on obesity and weight gain [20•]. These authors concluded that there is limited evidence that a longitudinal relationship exists between sedentary behavior, weight gain and risk of obesity. There was, however, reasonable level of evidence to conclude that sedentary behavior during childhood and adolescence is a strong predictor of obesity during adulthood [20•].

The purpose of the present study was to update previous reviews $\left[19,20^{\bullet}\right]$ to examine whether sitting time at baseline predicts weight gain at follow-up.

\section{Materials and Methods}

A literature search was conducted in the Medline database. The following keywords were used (MeSH, title words and text words) "cohort", "longitudinal", "prospectively", "prospective", "screen time", "screen", "sitting", "television", "TV", "weight gain", "obesity", "body mass index" and "overweight". All searches were limited to English-language peer-reviewed journal articles. The search strategy was conducted from January 2010 to August 2012 and involved screening reference lists of publications that matched the current inclusion criteria. Only full-text peer-reviewed articles were considered for inclusion. Titles and abstracts of the identified references were reviewed to exclude articles out of scope. Subsequently, full texts of all potentially relevant articles were read to come to the final selection.

The following inclusion criteria were used; the design had to be longitudinal (cause must precede effect); participants were healthy and non-pregnant at baseline; the dependent variable had to be a measure of weight gain (e.g., body weight, $\mathrm{BMI}$ ), and the exposure any measure of sedentary time. Studies reporting on longitudinal data from a control group in an intervention study were included, whereas all studies reporting on the effects of an intervention were excluded. Studies reporting on the effects of physical activity in general without reporting the effects of sedentary behavior were excluded. Of the 676 studies identified from the literature search, a total of 13 articles were identified and included in the review.

\section{Results}

Sample Characteristics

Samples sizes ranges from 254 to 120,877 . The mean age at baseline varied from 29 months to up around 52 years old. 
Seven of the studies were performed in children or adolescents (Table 1) whereas six studies were conducted in adults (Table 2). The follow-up period varied from 1 to 7.8 years in the studies including young people, and varied from 4 to 20 years in the studies including adults. Only one of the included studies examined whether sedentary time in adolescence predicted overweight in young adulthood [21].

\section{Measurements}

Except for two studies $[22,23 \bullet]$ all measurements on sedentary behavior were self- and/or parentally-reported. The majority of studies using self-report measures examined TV viewing $(\mathrm{n}=$ 11), in addition, some studies also included playing computer or video games $(n=2)$, computer use $(n=2)$, and total sitting time during the day $(n=2)$. Two of the studies conducted in the young population measured sedentary time by accelerometers, one study using cut-points of $<1100$ counts per minute (cpm) [22] and the other using a cut-point of $<100 \mathrm{cpm}$ [23•] to define total sedentary time. Nine of the studies reported results for objective measures of BMI, whereas four studies calculated BMI from self-reported height and weight. Other measures of weight gain were waist circumference $(n=4)$, hip circumference $(\mathrm{n}=1)$, waist-to-hip ratio $(\mathrm{n}=1)$, skinfold thickness $(\mathrm{n}=2)$, fat mass or percent body fat $(n=2)$.

\section{Young People}

In children and/or adolescents, the majority of studies $(n=6)$ found that higher levels of engagement in sedentary behavior was associated with increased risk of overweight/obesity $[21,24]$, increased BMI [23•, 25], or other indicators of fat mass [25-27] at follow-up. However, in three of the studies reporting an association, the analyses were not adjusted for the outcome measure at baseline $[21,23 \bullet, 27]$. In addition, in one of the studies, this association was found in boys but not in girls [26]. Similar findings were reported in another study, where computer time predicted increases in skinfold thickness in both boys and girls and increased BMI in boys, whereas time spent TV viewing predicted changes in BMI and hip circumference in boys, but not in girls [25]. One study found an association between increased sedentary behavior and increased percent body fat, but did not observe any association with BMI z-score [27]. The latter observation was supported by results from another study that also did not observe any association between sedentary time and BMI z-score [22]. One study [21] found that TV viewing in adolescence predicted overweight in young adulthood.

\section{Adults}

In adults, the results were mixed regarding the association between sitting time and weight gain. The majority of studies reported an association between sedentary behavior at baseline and weight gain $(\mathrm{n}=5)$, however, in three of these studies $[17,28,29]$ this association was attenuated toward the null after adjustment for baseline BMI and other covariates. One study reported that TV viewing at baseline was associated with weight gain 4 years later [30]. However, habitual active transport moderated the association between $\mathrm{TV}$-viewing and weight gain so that increased TV-viewing was associated with weight gain at follow-up among those who did not participate in everyday active transport. One study found no prospective associations between sitting time at baseline and weight gain at follow-up [31].

\section{Discussion}

This article appraised the peer-reviewed literature published between January 2010 and August 2012 that reported on the longitudinal relationship between sedentary time and weight gain in both young people and adults. The majority of the studies focused on a relationship between TV time or screen time and increased overweight, BMI or other indicators of fat mass. In the young population, mixed evidence was observed for a positive association between sedentary behavior at baseline and weight gain at follow-up. In adults, five studies reported that sedentary time at baseline predicted weight gain at follow-up, but in three of these studies the association that was no longer evident following adjustment for other variables, and one study reported no association between sedentary time and weight gain.

In longitudinal studies trying to decide temporal sequence and the direction of causality, one of the key components are that the cause precede the effect. In children and adolescents, several of the studies found a relationship between sedentary time and weight gain. However, in three of the studies reporting an association, the analyses were not adjusted for the outcome measure at baseline [21, 23•, 27]. When baseline measures are not accounted for, causality cannot be determined, suggesting that in these three studies it is not clear evidence that sedentary behavior leads to weight gain. In children and adolescents three studies found an association between sedentary behavior at baseline or change in sedentary behavior between baseline and followup and weight gain at follow-up, three studies reported an association but did not adjust for baseline variables and one study did not find any associations between increased sedentary behavior and increased weight gain. In those studies suggesting a significant association between sitting time and weight gain in several studies, the effect size was in general small or modest. Taken together, it appears premature to conclude there is convincing evidence for a prospective association between sedentary behavior and weight gain in the young population. This observation corroborates a 


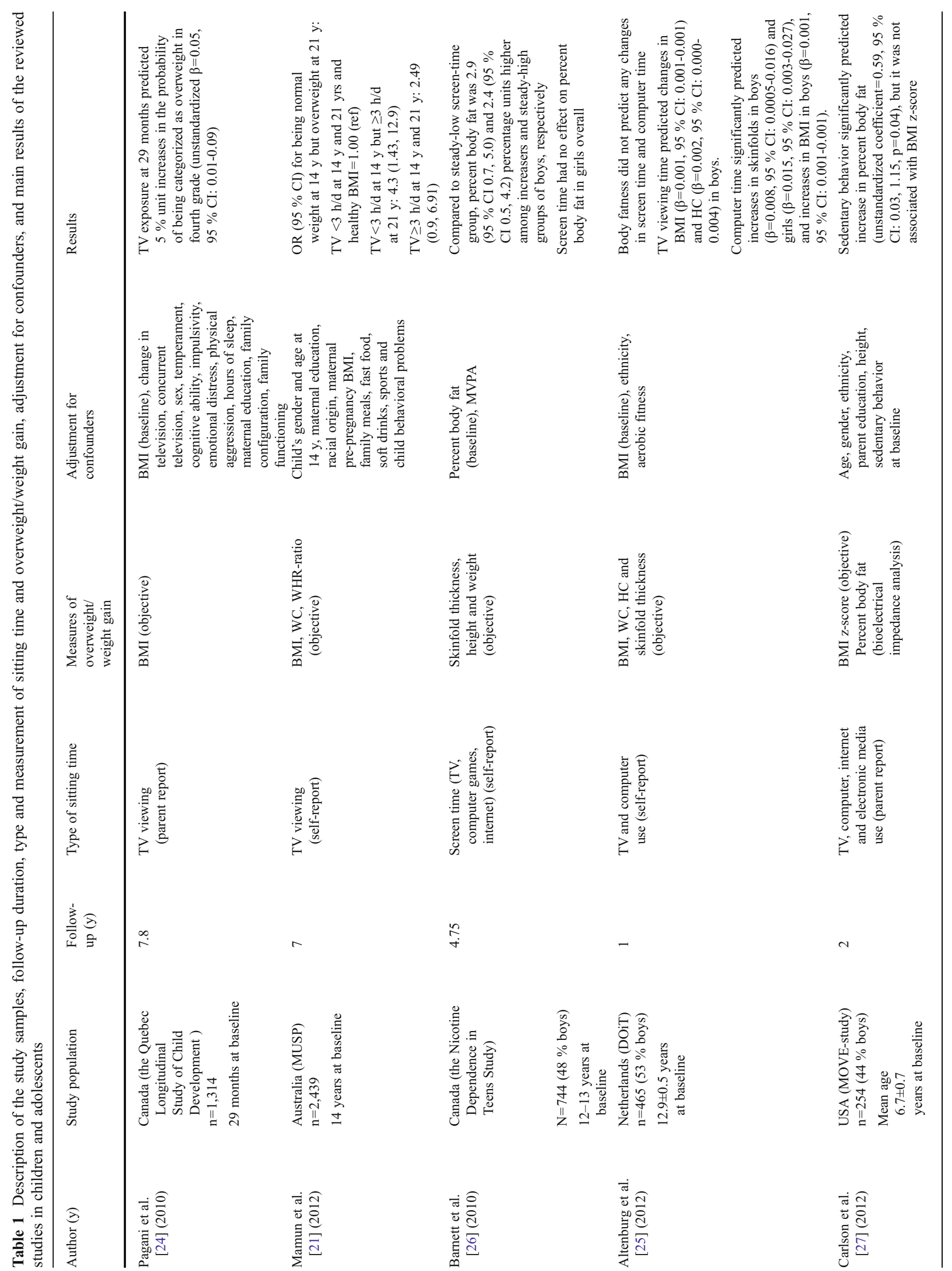


previous review concluding there was insufficient evidence for a longitudinal relationship between self- or proxy-reported sedentary time and indicators of fat mass [19].

Results from the studies in adults were somewhat mixed. Five of the studies reported significant associations between self-reported sedentary behavior at baseline with weight gain, but in three of these studies, these associations were no longer evident following adjustment for baseline BMI and other covariates [17, 28, 29]. Furthermore, one of the studies found the association to be moderated by habitual transport [30], meaning the association between TV viewing at baseline and weight gain at follow-up was only present among those who were inactive in everyday transport. In summary, two studies found that sedentary time at baseline predicted weight gain, whereas four studies did not report any associations. Therefore, it appears there is insufficient evidence that sedentary time is a strong predictor of weight gain in adults.

Explanations for the different findings could be the heterogeneity of the studies, which may reflect major differences in study designs, explanatory and outcome variables. Ten of the included studies adjusted their analyses for some form of physical activity. In adults, physical activity together with other covariates attenuated the association between sedentary behavior and weight gain in all studies but one [32•]. In children and adolescents, adjusting for physical activity attenuated the association in one study [22], and moderated the association in one study [26]. Some of the observed associations may be explained by residual confounding due to poorly measured or unmeasured confounders or bias due to misclassification. Different self-reported and objective measures of physical activity were used to assess physical activity as a confounder (MET hours per week, transport behavior, self-reported frequency of exercise, physical activity score, aerobic fitness, and MVPA), with some of these measures being rather crude. For example, in one study parents were asked to report the amount of time children spent on sports or exercise and they were categorized into "4-7 days per week" and "0-3 days per week" [21]. Further, energy intake is notoriously difficult to measure accurately and cannot be ruled out as a possible confounder. Even though six of the included studies attempted to adjust for energy intake, seven of the studies did not include this variable.

A common limitation of the studies reviewed was the measurement of sedentary behavior. The majority of the studies used self- or parent-reported sedentary time which can be affected by social desirability [33] and recall bias [34]. The majority of the studies using self-reported sedentary time addressed sitting during leisure time with a particular focus on TV-viewing time. Although brief self-reported questionnaires have shown to be adequate for group comparisons regarding TV viewing and computer use [35], 


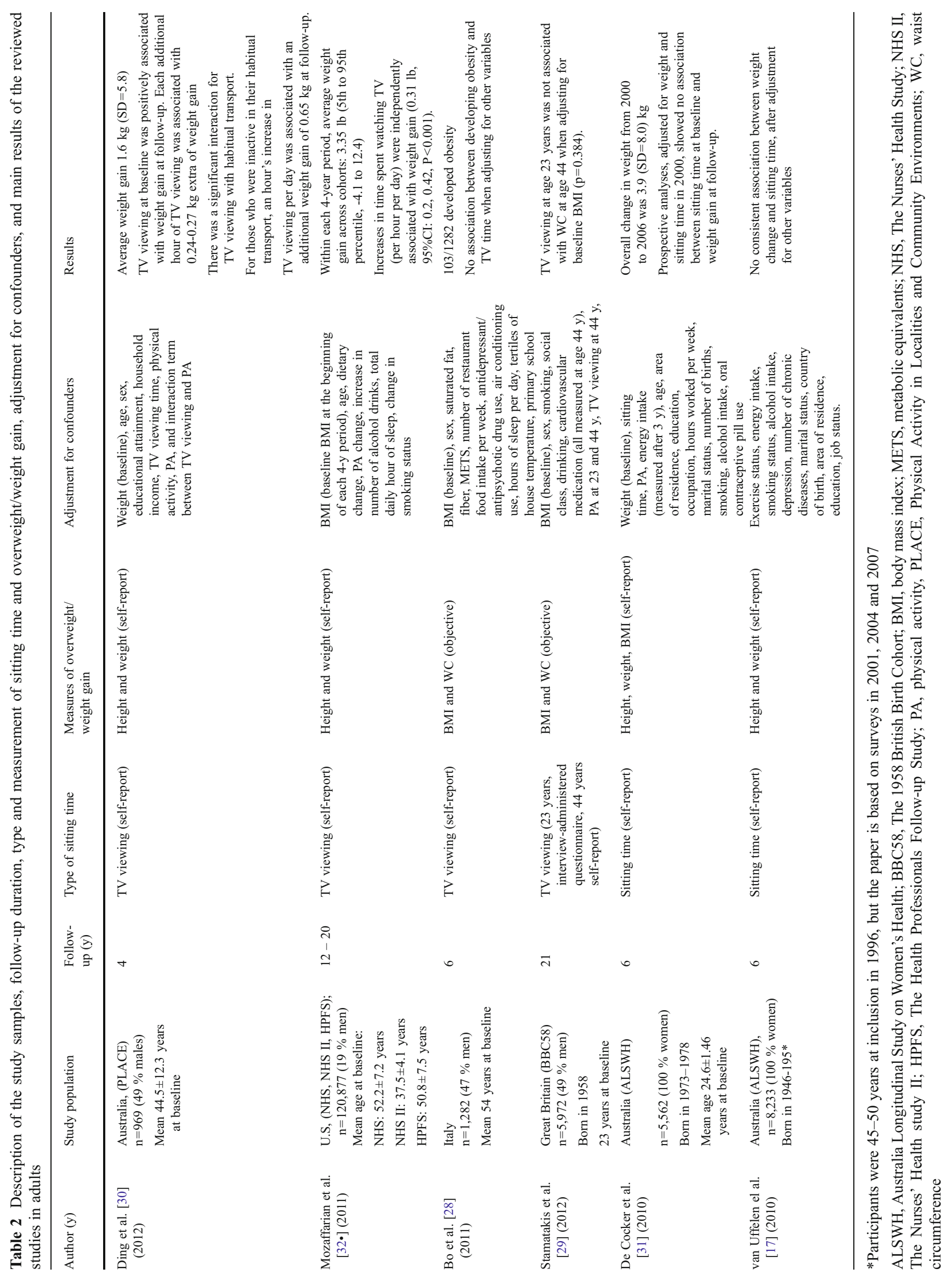


misclassification of self-reported sedentary time is unavoidable. However, if the error in the exposure variable is random it will attenuate the true association toward the null. Repeated measure of the exposure variable is recommended in future studies as this may decrease the chance of measurement error in the exposure. Only one of the included studies reported on the reliability and validity of the sitting time measure [30]. In adults, daily TV viewing were found to have excellent test-retest reliability (intraclass correlation coefficient $=0.82$ ) although the validity was relatively weak (Spearman's rank-order correlations with a 3 day activity $\log =0.3, \mathrm{p}<0.01)[30]$.

The association between self-reported and objectively measured sedentary time appears to be weak, indicating that the two measures are different constructs [11]. Only two studies, both in adolescents, used objective measures (i.e., accelerometers) to examine the associations between sedentary time and weight gain $[22,23 \bullet]$. These studies are less prone to the biases compared with self-report, and reduce the potential for differential measurement errors. However, some error may still be present. The uniaxial accelerometer predominantly captures ambulatory activities and cannot distinguish between different postures or variations in walking conditions. The findings in the two studies using accelerometers were inconsistent. While sedentary behavior was associated with greater increases in BMI at 50th percentile and above between ages 9 and 15 years [23•], no association was reported between sedentary behavior and increased BMI z-score from ages 7 to 9 years [22]. The inconclusive results might be due to the intensity thresholds used for sedentary behaviors as they differed by a factor of 10 between studies ( $<100 \mathrm{cpm}$ vs. $<1100 \mathrm{cpm}$ ). Unfortunately there is no consensus on the most appropriate threshold for identifying sedentary time from accelerometry. However, most studies have adopted a lower threshold (e.g., $<100 \mathrm{cpm}$ ). The difference between thresholds for sedentary time proposed is possibly explained by a combination of criterion methods (direct observation vs. indirect calorimetry) and the activities included in the calibration study when establishing the relationship between accelerometer counts and energy expenditure. The sedentary cut-point of $<100 \mathrm{cpm}$, provides a useful estimate of sitting time, however, some standing time may also have been included. A cut-point of $<1100 \mathrm{cpm}$ appears to be fairly high, and will most likely also include activities of light intensity. This may therefore explain why the latter study did not observe an association between sedentary time and weight gain.

The majority of the studies included in the review measured sedentary behavior and weight at the same time points with no intermediate measure of sedentary behavior. Some of the studies examined the association between change in exposure between baseline and follow-up with change in the outcome, but such analytical model cannot determine the direction of association. Other common methodological limitations in the studies reviewed include limited information on participation rate and selective non-response at follow-up. Some of the studies that did report attrition, reported considerable loss to follow-up (approximately $60 \%)[21,30]$ which may also bias the observed results.

TV viewing was the most frequent surveyed type of sedentary behavior in both youth and adults. Watching TV is the predominant leisure time sedentary behavior in adults with averaging more than 3 hours in UK, the US and Australia [36-38]. However, even though TV does occupy considerable amounts of sedentary time, other domainspecific sitting behaviors such as occupational sitting, using the computer, playing electronic games, reading, talking on the telephone and travelling by bus, car or train also contribute to people's sedentary time, not captured in most of the reviewed studies. TV viewing time appears not to be a good marker of overall sedentary time in young people [39]. Further, the possibility of residual confounding on the association between TV viewing and weight gain cannot be excluded [11]. TV viewing appears to be associated with snacking both during viewing and at other times [40-42]. Also, food advertisement on TV might promote an unhealthy diet [43] suggesting that some of the reported associations may be confounded by diet. TV viewing may also be a marker for an unhealthy lifestyle in general. In addition, reverse causation cannot be completely ruled out in any observational research, as those who are more obese at the inception of the study may report higher amounts of sedentary time. Three of the studies in the review did also examine reverse causality $[17,25,31]$. However, none of the studies reported prospective relationships between weight and change in sitting time in neither adults [31] nor adolescents [25]. This is in contrast to some emerging evidence that higher levels of adiposity at baseline predicts higher amounts of sedentary time at follow-up in children [44, 45] and higher levels of physical inactivity in adults [46].

Results on the association between sedentary behavior at baseline and weight gain at follow-up are still inconclusive. Further studies with objective measures of sitting time and physical activity are required to clarify the direction of the relationship between sedentary behavior and weight gain. This is important as it is still unknown what type of intervention is most beneficial in preventing the increases in overweight and obesity in the population.

\section{Conclusions}

Findings from this review suggest that there is mixed evidence for a positive relationship between sedentary behavior and weight gain in children and adolescents, and there is insufficient evidence for a relationship between sedentary 
behavior and weight gain in adults. To better be able to understand the causal relationship and direction of association between sedentary behavior and weight gain, more high-quality studies using repeated objective measures of sitting time and physical activity is warranted. Appropriate adjustment for baseline measures of the outcome and potential confounders are highly recommended.

Disclosure No potential conflicts of interest relevant to this article were reported.

\section{References}

Papers of particular interest, published recently, have been highlighted as:

- Of importance

1. Hansen BH, Kolle E, Dyrstad SM, Holme I, Anderssen SA. Accelerometer-determined physical activity in adults and older people. Med Sci Sports Exerc. 2012;44:266-72.

2. Hagstromer M, Oja P, Sjostrom M. Physical activity and inactivity in an adult population assessed by accelerometry. Med Sci Sports Exerc. 2007;39:1502-8.

3. Pate RR, O'Neill JR, Lobelo F. The evolving definition of "sedentary". Exerc Sport Sci Rev. 2008;36:173-8.

4. Owen N, Healy GN, Matthews CE, Dunstan DW. Too much sitting: the population health science of sedentary behavior. Exerc Sport Sci Rev. 2010;38:105-13.

5. Wijndaele $\mathrm{K}$, Brage $\mathrm{S}$, Besson $\mathrm{H}$, et al. Television viewing and incident cardiovascular disease: prospective associations and mediation analysis in the EPIC Norfolk Study. PLoS One. 2011;6: e20058.

6. Patel AV, Bernstein L, Deka A, et al. Leisure time spent sitting in relation to total mortality in a prospective cohort of US adults. Am J Epidemiol. 2010;172:419-29.

7. Dunstan DW, Barr EL, Healy GN, et al. Television viewing time and mortality: the Australian Diabetes, Obesity and Lifestyle Study (AusDiab). Circulation. 2010;121:384-91.

8. van der Ploeg HP, Chey T, Korda RJ, Banks E, Bauman A. Sitting time and all-cause mortality risk in 222497 Australian adults. Arch Intern Med. 2012;172:494-500.

9. Veerman JL, Healy GN, Cobiac LJ, et al. Television viewing time and reduced life expectancy: a life table analysis. Br J Sports Med. 2012;46:927-30.

10. Grontved A, Hu FB. Television viewing and risk of type 2 diabetes, cardiovascular disease, and all-cause mortality: a metaanalysis. JAMA. 2011;305:2448-55.

11. Ekelund U. Commentary: Too much sitting - a public health threat? Int J Epidemiol. 2012;41:1353-5.

12. Stamatakis E, Hamer M, Tilling K, Lawlor DA. Sedentary time in relation to cardio-metabolic risk factors: differential associations for self-report vs accelerometry in working age adults. Int J Epidemiol. 2012;41:1328-37.

13. Manini TM, Everhart JE, Patel KV, et al. Daily activity energy expenditure and mortality among older adults. JAMA. 2006;296:171-9.

14. Buchman AS, Yu L, Boyle PA, Shah RC, Bennett DA. Total daily physical activity and longevity in old age. Arch Intern Med. 2012;172:444-6.
15. Jakes RW, Day NE, Khaw KT, et al. Television viewing and low participation in vigorous recreation are independently associated with obesity and markers of cardiovascular disease risk: EPICNorfolk population-based study. Eur J Clin Nutr. 2003;57:108996.

16. Kronenberg F, Pereira MA, Schmitz MK, et al. Influence of leisure time physical activity and television watching on atherosclerosis risk factors in the NHLBI Family Heart Study. Atherosclerosis. 2000;153:433-43.

17. van Uffelen JG, Watson MJ, Dobson AJ, Brown WJ. Sitting time is associated with weight, but not with weight gain in mid-aged Australian women. Obesity (Silver Spring). 2010;18:1788-94.

18. Prentice-Dunn H, Prentice-Dunn S. Physical activity, sedentary behavior, and childhood obesity: a review of cross-sectional studies. Psychol Health Med. 2012;17:255-73.

19. Chinapaw MJ, Proper KI, Brug J, van Mechelen W, Singh AS. Relationship between young peoples' sedentary behaviour and biomedical health indicators: a systematic review of prospective studies. Obes Rev. 2011;12:e621-32.

20. - Thorp AA, Owen N, Neuhaus M, Dunstan DW. Sedentary behaviors and subsequent health outcomes in adults a systematic review of longitudinal studies, 1996-2011. Am J Prev Med. 2011;41:207-15. This is a systematic review reporting on the relationship between sedentary behavior with health-related outcomes in adults.

21. Mamun AA, O'Callaghan MJ, Williams G, Najman JM. Television watching from adolescence to adulthood and its association with BMI, waist circumference, waist-to-hip ratio and obesity: a longitudinal study. Public Health Nutr. 2012;1:11.

22. Basterfield L, Pearce MS, Adamson AJ, et al. Physical activity, sedentary behavior, and adiposity in English children. Am J Prev Med. 2012;42:445-51.

23. - Mitchell JA, Pate RR, Beets MW, Nader PR. Time spent in sedentary behavior and changes in childhood BMI: a longitudinal study from ages 9 to 15 years. Int J Obes (Lond). 2012. This study is important due to the use of objective measures of both sedentary time and weight gain in a cohort of adolescents.

24. Pagani LS, Fitzpatrick C, Barnett TA, Dubow E. Prospective associations between early childhood television exposure and academic, psychosocial, and physical well-being by middle childhood. Arch Pediatr Adolesc Med. 2010;164:425-31.

25. Altenburg TM, Singh AS, van Mechelen W, Brug J, Chinapaw MJ. Direction of the association between body fatness and self-reported screen time in Dutch adolescents. Int J Behav Nutr Phys Act. 2012;9:4.

26. Barnett TA, O'Loughlin J, Sabiston CM, et al. Teens and screens: the influence of screen time on adiposity in adolescents. Am J Epidemiol. 2010;172:255-62.

27. Carlson JA, Crespo NC, Sallis JF, Patterson RE, Elder JP. Dietaryrelated and physical activity-related predictors of obesity in children: a 2-year prospective study. Child Obes. 2012;8:110-5.

28. Bo S, Ciccone G, Durazzo M, et al. Contributors to the obesity and hyperglycemia epidemics. A prospective study in a populationbased cohort. Int J Obes (Lond). 2011;35:1442-9.

29. Stamatakis E, Hamer M, Mishra GD. Early adulthood television viewing and cardiometabolic risk profiles in early middle age: results from a population, prospective cohort study. Diabetologia. 2012;55:311-20.

30. Ding D, Sugiyama T, Owen N. Habitual active transport, TV viewing and weight gain: a four year follow-up study. Prev Med. 2012;54:201-4.

31. De Cocker KA, van Uffelen JG, Brown WJ. Associations between sitting time and weight in young adult Australian women. Prev Med. 2010;51:361-7.

32. - Mozaffarian D, Hao T, Rimm EB, Willett WC, Hu FB. Changes in diet and lifestyle and long-term weight gain in women and men. 
N Engl J Med. 2011;364:2392-404. This study study provide a description of how specific dietary and lifestyle factors are independently associated with long term weight-gain in a large cohort of adults.

33. Adams SA, Matthews CE, Ebbeling CB, et al. The effect of social desirability and social approval on self-reports of physical activity. Am J Epidemiol. 2005;161:389-98.

34. Sallis JF, Saelens BE. Assessment of physical activity by selfreport: status, limitations, and future directions. Res Q Exerc Sport. 2000;71:S1-S14.

35. Schmitz KH, Harnack L, Fulton JE, et al. Reliability and validity of a brief questionnaire to assess television viewing and computer use by middle school children. J Sch Health. 2004;74:370-7.

36. Office for National Statistics. The Time Use Survey, 2005. How We Spend Our Time. London: Office for National Statistics; 2006.

37. Nielsen Media Research. Nielsen Report on Television. New York: Nielsen Media Research; 2000.

38. Australian Bureau of Statistics. How Australians Use Their Time, 2006. Canberra: Commonwealth of Australia; 2008.

39. Biddle SJ, Gorely T, Marshall SJ. Is television viewing a suitable marker of sedentary behavior in young people? Ann Behav Med. 2009;38:147-53.
40. Blass EM, Anderson DR, Kirkorian HL, Pempek TA, Price I, Koleini MF. On the road to obesity: television viewing increases intake of high-density foods. Physiol Behav. 2006;88:597-604.

41. Vereecken CA, Todd J, Roberts C, Mulvihill C, Maes L. Television viewing behaviour and associations with food habits in different countries. Public Health Nutr. 2006;9:244-50.

42. Utter J, Scragg R, Schaaf D. Associations between television viewing and consumption of commonly advertised foods among New Zealand children and young adolescents. Public Health Nutr. 2006;9:606-12.

43. Harris JL, Bargh JA, Brownell KD. Priming effects of television food advertising on eating behavior. Health Psychol. 2009;28:404-13.

44. Ekelund U, Luan J, Sherar LB, Esliger DW, Griew P, Cooper A. Moderate to vigorous physical activity and sedentary time and cardiometabolic risk factors in children and adolescents. JAMA. 2012;307:704-12.

45. Kwon S, Janz KF, Burns TL, Levy SM. Effects of adiposity on physical activity in childhood: Iowa Bone Development Study. Med Sci Sports Exerc. 2011;43:443-8.

46. Golubic R, Ekelund U, Wijndaele $\mathrm{K}$ et al. Rate of weight gain predicts change in physical activity levels: a longitudinal analysis of the EPIC-Norfolk cohort. Int J Obes (Lond). 2012. 